\title{
Systemic lupus erythematosus flare triggered by a mosquito bite: the first case report
}

This article was published in the following Dove Press journal:

Open Access Rheumatology: Research and Reviews

\section{Vadood Javadi Parvaneh \\ Mohsen Jari \\ Sheri Motahari \\ Khosro Rahmani \\ Reza Shiari}

Department of Pediatric Rheumatology, Shahid Beheshti University of Medical

Sciences, Tehran, Iran

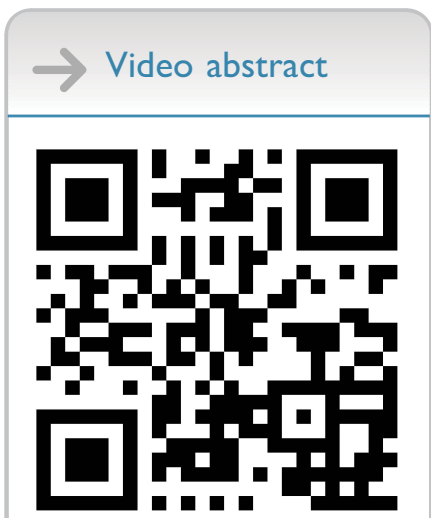

Point your SmartPhone at the code above. If you have a QR code reader the video abstract will appear. Or use: https://youtu.be/S6CwhiveaEw
Correspondence: Reza Shiari

Department of Pediatric Rheumatology, Shahid Beheshti University of Medical Sciences, Faculty of Medicine, Mofid

Children's Hospital, Shariati Avenue,

Tehran, Iran

Tel +98212 2227033

Fax +982122227033

Email shiareza@yahoo.com
Introduction: Systemic lupus erythematosus (SLE) is a chronic autoimmune disease with a wide, various, and sometimes deceptive clinical and serological manifestations. Environmental factors such as ultraviolet radiation, viral infections, drugs, hormones, and chemicals could trigger SLE flares in genetically predisposed patients.

Case report: We presented a 13-year-old girl with the first presentation of systemic lupus erythematosus triggered by a mosquito bite. She presented with a malar rash started after a mosquito bite on her left cheek. She had oral ulcers, photosensitivity, lymphopenia, proteinuria, and positive serologic tests for SLE. Renal biopsy revealed class II lupus nephritis.

Conclusion: Environmental factors can trigger the onset of SLE in genetically susceptible cases. Besides microbial agents, UV radiation, hormones, drugs, emotional stresses, immunization, and chemicals are some of the published examples. We presented a case with a mosquito bite as the possible environmental trigger.

Keywords: mosquito bite, systemic lupus erythematosus, children, pediatrics, etiology, case report

\section{Introduction}

Systemic lupus erythematosus (SLE) is a multisystem autoimmune disease with circulating autoantibodies against self-antigens. ${ }^{1,2}$ The etiology and pathogenesis of SLE is complex and poorly understood. It is proposed to be a combination of immunological abnormalities, genetic, hormones, environmental factors, ultraviolet radiation, viral infection, and drugs. Several environmental triggers have been identified. $^{3-5}$ Herein, we presented a case with lupus triggered by a mosquito bite.

\section{Case report}

A 13-year-old girl presented to a pediatric clinic with a history of erythematous facial rash on her cheeks and the bridge of the nose. This rash was started two weeks ago after a mosquito bite on her left cheek. It became red and swollen first, without any sign of infection or cellulitis at or around the bite site. Finally, she was referred to our hospital for further evaluation. On physical examination, she had malar rash, oral ulcers, and history of photosensitivity. In the site of the mosquito bite, there was a papule with a central hole. For further investigation, she was admitted to the hospital. The patient had oral ulcers, photosensitivity, malaise, weight loss, and vague musculoskeletal pain for a couple of months. Laboratory tests revealed lymphopenia (white blood cell count $4,300 / \mathrm{mm}^{3}$, absolute lymphocyte count: $903 / \mathrm{mm}^{3}$ ) and positive antinuclear antibody (1/320 titer). Erythrocyte sedimentation rate and C-reactive protein were within the 
normal limits. Anti-double-stranded DNA (ds-DNA) was positive [ds-DNA: $98 \mathrm{mg} / \mathrm{dl}(\mathrm{N}<24)]$. Urine analysis demonstrated urine protein $2+$ and the urine random $\mathrm{Pr} / \mathrm{Cr}$ ratio was 0.45. Complement levels were low [C3:65.3 mg/dl $(\mathrm{N}=90-180), \mathrm{C} 4: 7.6 \mathrm{mg} / \mathrm{dl}(\mathrm{N}=10-40)]$. Liver and thyroid function tests and creatinine level were normal. Based on her physical exams and laboratory tests, the patient was diagnosed as SLE and underwent on renal biopsy. Then treatment with methylprednisolone $(30 \mathrm{mg} / \mathrm{kg} /$ day for three consecutive days) started and subsequently changed to oral prednisolone (2 $\mathrm{mg} / \mathrm{kg} / \mathrm{day})$. Hydroxychloroquine and Mycophenolate mofetil were also prescribed. Four weeks later on follow up visit, her condition improved and oral ulcers healed, also her laboratory tests such as white blood cell count, anti-double-strand DNA (ds- DNA) antibody and complement levels became normal. Urine analysis showed 1 + protein and Urine random $\mathrm{Pr} / \mathrm{Cr}$ ratio was 0.3 . Renal biopsy revealed class II lupus nephritis, according to International Society of Nephrology/Renal Pathology Society classification. . Her treatment was continued as the same. On follow up visit till three months, the patient was in good condition and her physical examination and laboratory tests were normal.

\section{Discussion}

The girl presented with malar rash, photosensitivity, oral ulcers, lymphopenia, proteinuria (and later ISN/RPS class II lupus nephritis in renal histology), low complements, positive ANA and positive anti-ds-DNA antibodies. Therefore, the diagnosis of SLE was confirmed. She had a history of a mosquito bite on her cheek which became red and swollen without any manifestation of infection such as pain, tenderness, and warmness. The rash had been spread on her face. On presentation, she had a malar rash with butterfly distribution and a papule with a central hole in it. So, according to the classification of cutaneous signs in patients with lupus erythematosus (LE), the malar rash and a history of photosensitive lupus rash were under the category of specific signs of dermoepidermal LE (acute cutaneous lupus erythematosus).

Systemic lupus erythematosus (SLE) is a chronic multisystemic autoimmune disease. The pathogenesis of SLE is the results from the complex interaction between genetic, epigenetic, hormonal factors, and environmental exposures. ${ }^{1,2}$ The environment probably has a role in the etiology of SLE via its effect on the immune system. ${ }^{3-5}$

A dysregulated inflammatory response- including innate, cellular, and humoral immune system- leads to the overproduction of autoantibodies. This is a result of the interplay between genetic susceptibility, environmental factors, and hormonal influence. The genes involved play a role in immune system regulation. ${ }^{3}$

Environmental factors such as infections -especially viral infections such as Epstein-Barr virus (EBV), Cytomegalovirus (CMV), and Parvovirus B19-have been associated with the pathogenesis of the disease in genetically predisposed patients. ${ }^{4,5}$ These viruses can induce autoimmunity through molecular mimicry, activation of $\mathrm{T}$ lymphocytes by super-antigens, and bystander activation. $^{4-7}$

Ultraviolet (UV) radiation can be involved in the pathogenesis of the cutaneous as well as SLE through induction of apoptosis, DNA breakage, and exposing it to the immune system. UV radiation can also decrease methylation which may alter gene expression too. ${ }^{8}$ The possible association of vaccination with SLE has been reported in some case reports especially after hepatitis B, influenza and human papillomavirus. Vaccination can induce adjuvant-induced autoimmunity. ${ }^{9}$ The predominance of SLE in female patients between menarche and menopause and SLE flare in pregnancy, oral contraceptives, and hormone therapy are evidence of a role of estrogens and low levels of androgens in disease risk. Large declines in vitamin D (for example winter and autumn as low daylight months) can trigger lupus flares. Flare rate about $61 \%$ in psychological stress was reported in one study. More than 80 medications have been associated with drug-induced lupus erythematosus with antihistone antibodies. Also, some drugs could exacerbate underlying SLE. Smoking, pesticides, solvents, silica, heavy metals, and hair dyes have been associated with SLE with varying degrees of evidence. Therefore, some of the environmental factors could trigger SLE flares such as ultraviolet radiation, viral infections, drugs, hormones, and chemicals. ${ }^{10-12}$

\section{Conclusion}

Lupus flare is a measurable increase in disease activity in one or more organ systems involving new or worse clinical signs and symptoms and/or laboratory measurements. Our patient was in good condition until she was bitten by a mosquito. We hypothesize that the mosquito bite in susceptible genetically patient could have provoked some of the aforementioned mechanisms, resulting in ongoing inflammation and triggering lupus to flare-up. To our 
knowledge, this case is the first reported lupus flare triggered by a mosquito bite.

\section{Consent form}

We confirm that the written informed consent form has been provided by the parents to have the case details published. Also, we restate that institutional approval is not required to publish the case details.

\section{Acknowledgment}

This research did not receive any specific grant from funding agencies in the public, commercial, or not-forprofit sectors.

\section{Disclosure}

The authors report no conflicts of interest in this work.

\section{References}

1. Belot A, Cimaz R. Monogenic forms of systemic lupus erythematosus: new insights into SLE pathogenesis. Pediatr Rheumatol. 2012;10 (1):21. doi:10.1186/1546-0096-10-24

2. Schwartzman-Morris J, Putterman C. Gender differences in the pathogenesis and outcome of lupus and of lupus nephritis. Clin Dev Immunol. 2012;2012:1-9. doi:10.1155/2012/604892

3. Sag E, Tartaglione A, Batu ED, et al. Performance of the new SLICC classification criteria in childhood systemic lupus erythematosus: a multicentre study. Clin Exp Rheumatol. 2014;32(3):440-444.
4. Crispín JC, Liossis SN, Kis-Toth K, et al. Pathogenesis of human systemic lupus erythematosus: recent advances. Trends $\mathrm{Mol} \mathrm{Med.}$ 2010;16(2):47-57. doi:10.1016/j.molmed.2009.12.005

5. Esposito S, Bosis S, Semino M, Rigante D. Infections and systemic lupus erythematosus. Eur J Clin Microbiol Infect Dis. 2014;33 (9):1467-1475. doi:10.1007/s10096-014-2098-7

6. Cooper GS, Gilbert KM, Greidinger EL, et al. Recent advances and opportunities in research on lupus: environmental influences and mechanisms of disease. Environ Health Perspect. 2008;116 (6):695-702. doi:10.1289/ehp.11092

7. Rigante D, Mazzoni MB, Esposito S. The cryptic interplay between systemic lupus erythematosus and infections. Autoimmun Rev. 2014;13(2):96-102. doi:10.1016/j.autrev.2013.09.004

8. Wang GS, Zhang M, Li XP, et al. Ultraviolet B exposure of peripheral blood mononuclear cells of patients with systemic lupus erythematosus inhibits DNA methylation. Lupus. 2009;18(12):1037-1044. doi:10.1177/0961203309106181

9. Perricone C, Colafrancesco S, Mazor RD, Soriano A, Agmon-Levin N, Shoenfeld Y. Autoimmune/inflammatory syndrome induced by adjuvants (ASIA) 2013: unveiling the pathogenic, clinical and diagnostic aspects. $J$ Autoimmun. 2013;47:1-6. doi:10.1016/j. jaut.2013.10.004

10. Roussou E, Iacovou C, Weerakoon A, Ahmed K. Stress as a trigger of disease flares in SLE. Rheumatol Int. 2013;33(5):1367-1370. doi:10.1007/s00296-011-2292-1

11. Caldas CA, de Carvalho JF. The role of environmental factors in the pathogenesis of non-organ-specific autoimmune diseases. Best Pract Res Clin Rheumatol. 2012;26(1):5-11. doi:10.1016/j. berh.2012.01.010

12. Zandman-Goddard G, Solomon M, Rosman Z, Peeva E, Shoenfeld Y. Environment and lupus-related diseases. Lupus. 2012;21(3):241-250. doi:10.1177/0961203311426568

\section{Publish your work in this journal}

Open Access Rheumatology Research and Reviews is an international, peer-reviewed, open access journal publishing original research, reports, editorials, reviews and commentaries on all aspects of clinical and experimental rheumatology in the clinic and laboratory including the following topics: Pathology, pathophysiology of rheumatological diseases; Investigation, treatment and managemen of rheumatological diseases; Clinical trials and novel pharmacological approaches for the treatment of rheumatological disorders. The manuscript management system is completely online and includes a very quick and fair peer-review system, which is all easy to use. Visit http://www.dovepress.com/testimonials.php to read real quotes from published authors. 